Pacific Journal of Mathematics

SPECTRAL SYMMETRY OF THE DIRAC OPERATOR FOR 


\title{
SPECTRAL SYMMETRY OF THE DIRAC OPERATOR FOR COMPACT AND NONCOMPACT SYMMETRIC PAIRS
}

\author{
H. D. Fegan, B. Steer, ANd L. Whiteway
}

The aim of this paper is to prove a vanishing of theorem for the Dirac operator on a symmetric pair. In fact, we prove a stronger result: that the Dirac operator has spectral $G$-symmetry.

THEOREM 1.1. Let $(G, K)$ be a symmetric pair of rank two or greater, of compact or noncompact type and $\Gamma \subset G$ a co-compact discrete subgroup. Let $\rho$ be a metric on $\Gamma \backslash G$ whose lift to $G$ is $G$-left and $K$-right invariant. Then, the Dirac operator has spectral G-symmetry: that is, for each eigenvalue $\lambda$ the eigenspace $V_{\lambda}$ is $G$-isomorphic to the eigenspace $V_{-\lambda}$.

COROLlaRY 1.2. The equivariant $\eta$-function vanishes identically: $\eta_{G}(s, g)=0$.

The importance of the eta invariant and questions of spectral symmetry has long been recognized, see [1]. If $\operatorname{dim} G \neq 4 k+3$, the spectrum is symmetric for algebraic reasons. However, as the example in [4] shows, this spectrum need not be symmetric if $\operatorname{dim} G=3$. For an odd dimensional simply connected Lie group with bi-invariant metric, the map $x \mapsto x^{-1}$ is an orientation reversing isometry and we again get spectral symmetry. However, this map may well not descend to quotients $\Gamma \backslash G$; for example, we know the spectrum for $\mathrm{SO}(3) \cong \mathrm{SU}(2) /\{ \pm 1\}$ is not symmetric. Furthermore, if $G$ is a noncompact rank one group and $\Gamma$ a co-compact discrete subgroup then, with respect to certain natural metrics on $\Gamma \backslash G$, the spectrum fails to be symmetric, see [6]. Thus, the result does not hold in the rank one case.

In $\S 2$ we discuss the case of a symmetric pair of compact type. This is done in some detail. Section 3 contains the case of noncompact type. Since this is similar to the compact type, we concentrate on presenting the changes in the new case. We do not consider the case of a symmetric pair of Euclidean type. 
The first author was supported by an Efroymson Memorial lectureship.

2. Spectral symmetry for a symmetric pair of compact type. Let $(G, K)$ be a symmetric pair of compact type. Then the Lie algebra of $G$ decomposes as $\mathscr{G}=\mathscr{K} \otimes \mathscr{P}$ with bracket relations $[\mathscr{K}, \mathscr{K}] \subset$ $\mathscr{K},[\mathscr{K}, \mathscr{P}] \subset \mathscr{P}$ and $[\mathscr{P}, \mathscr{P}] \subset \mathscr{K}$. With respect to the negative of the Killing form let $E_{1}, \ldots, E_{r}$ be an orthonormal basis for $\mathscr{K}$ and $E_{r+1}, \ldots, E_{r+s}$ one for $\mathscr{P}$ so $r+s=\operatorname{dim} G$ is odd. Throughout this and the following section we shall use the following convention: Latin subscripts run from 1 to $r$ and Greek subscripts from $r+1$ to $r+s$. Let $t>0$ be a real parameter and set $e_{i}=E_{i} / t$ and $e_{\alpha}=E_{\alpha}$. Let $\rho_{t}$ denote the left invariant metric such that $e_{1}, \ldots, e_{r+s}$ is an orthonormal basis of $\mathscr{G}$. Thus for $t \neq 1 \rho_{t}$ is $G$ left-invariant but only $K$ right-invariant. The effect is to scale the metric on the fibers and leave it unchanged on the base of the fibration $K \rightarrow G \rightarrow G / K$. Further set $w_{t}=e^{q} \gamma_{1}, \ldots, e_{r+s}$ where $q=(r+s+1)(r+s+2) / 2$ and let $\psi^{t}$ denote a basic spinor corresponding to the $e_{1}, \ldots, e_{r+s}$ basis. When $t=1$ the subscript $t$ will be omitted. There is a canonical isomorphism between the Clifford algebra associated to $\rho$ and that associated to $\rho_{t}$. Under this isomorphism $e_{i}$ is the image of $E_{i}, e_{\alpha}$ the image of $E_{\alpha}$ and $\psi^{t}$ that of $\psi$. Using this isomorphism, we notice that (with $1 \leq i_{j} \leq r+s$ )

$$
e_{e_{1}} \cdots e_{i_{k}} \psi^{t}=E_{i_{1}} \cdots E_{i_{k}} \psi
$$

for any set of basis vectors, where the Clifford product on the lefthand side is relative to the $\rho_{t}$ but on the right-hand side is relative to $\rho=\rho_{1}$. This same isomorphism is used implicitly in later expressions.

The Dirac operator is

$$
P_{t}=\sum \omega_{t} e_{i} \nabla_{e_{t}}^{t}+\sum \omega_{t} e_{\alpha} \nabla_{e_{\alpha}}^{t}
$$

where $\nabla^{t}$ is the Levi-Civita connection corresponding to $\rho_{t}$. We can identify the space of sections $\Gamma \underset{\sim}{S})$ with $C_{\infty}(\Gamma \backslash G) \otimes S$ using left translation. Then for a basic spinor $\psi^{t}=1 \otimes s^{t}$

$$
\begin{aligned}
P_{t}\left(f \otimes s^{t}\right)= & \sum \nu\left(e_{i}\right) f \otimes \omega_{t} e_{i} s^{t}+\sum \nu\left(e_{\alpha}\right) f \otimes \omega_{t} e_{\alpha} s^{t} \\
& +f P_{t}\left(1 \otimes s^{t}\right) \\
= & \frac{1}{t} \sum \nu\left(E_{i}\right) f \otimes \omega E_{s} s+\sum \nu\left(E_{\alpha}\right) f \otimes \omega E_{\alpha} s \\
& +f P_{t}\left(1 \otimes s^{t}\right) .
\end{aligned}
$$


If we define $Q_{K}=\sum \nu\left(E_{i}\right) \otimes \omega E_{i}, Q_{P}=\sum \nu\left(E_{\alpha}\right) \otimes \omega E_{\alpha}$ and $Q_{t}=$ $1 / t Q_{K}+Q_{P}$ then we see that

$$
P_{t}\left(f \otimes s^{t}\right)=Q_{t}(f \otimes s)+f P_{t}\left(1 \otimes s^{t}\right) .
$$

Thus it remains to calculate $P_{t} \psi^{t}$. First we calculate $\nabla^{t}$.

Proposition 2.1. (i) $\nabla_{e_{i}}^{t} e_{j}=\left(1 / t^{2}\right) \nabla_{E_{i}} E_{j}$,

(ii) $\nabla_{e_{i}}^{t} e_{\beta}=(2 / t-t) \nabla_{E_{i}} E_{\beta}$,

(iii) $\nabla_{e_{\alpha}}^{t} e_{j}=t \nabla_{E_{\alpha}} E_{j}$,

(iv) $\nabla_{e_{\alpha}}^{t^{\alpha}} e_{\beta}=\nabla_{E_{\alpha}} E_{\beta}$.

Proof. These follow from the following formulae:

$$
\begin{aligned}
\text { (i) }\left\langle\nabla_{e_{i}}^{t} e_{j}, e_{k}\right\rangle_{t}=\frac{1}{t}\left\langle\nabla_{E_{i}} E_{j}, E_{k}\right\rangle, \\
\text { (ii) }\left\langle\nabla_{e_{i}}^{t} e_{\beta}, e_{\gamma}\right\rangle_{t}=(2 / t-t)\left\langle\nabla_{E_{i}} E_{\beta}, E_{\gamma}\right\rangle, \\
\text { (iii) }\left\langle\nabla_{e_{\alpha}}^{t} e_{j}, e_{\gamma}\right\rangle_{t}=t\left\langle\nabla_{E_{\alpha}} E_{j}, E_{\gamma}\right\rangle, \\
\text { (iv) }\left\langle\nabla_{e_{\alpha}}^{t} e_{\beta}, e_{k}\right\rangle_{t}=t\left\langle\nabla_{E_{\alpha}} E_{\beta}, E_{k}\right\rangle,
\end{aligned}
$$

and the observation that all similar expressions with an odd number of Greek subscripts are zero. These formulae use the notation $\langle,\rangle_{t}$ for the inner product given by $\rho_{t}$. The calculations are similar to those of [3]. In obtaining these formulae, we use the fact that ad $E_{i}$ (for $1 \leq i \leq r+s$ ) is $\rho_{t}$-skew. For orthonormal left invariant vector fields $X, Y$ and $Z$ there is the formula

$$
\left\langle\nabla_{X} Y, Z\right\rangle=\frac{1}{2}(\langle Z,[X, Y]\rangle-\langle Y,[X, Z]\rangle-\langle X,[Y, Z]\rangle) .
$$

From this, we see $\nabla_{E_{t}} E_{j}=\frac{1}{2}\left[E_{i}, E_{j}\right]$, which is also useful.

From [2] $\chi(X)=-\frac{1}{4} \sum\left[X, E_{i}\right] E_{i}-\frac{1}{4} \sum\left[X, E_{\alpha}\right] E_{\alpha}$. We make the following definitions:

$$
\begin{aligned}
\chi_{K}(X) & =-\frac{1}{4} \sum\left[X, E_{i}\right] E_{i}, \\
\chi_{P}(X) & =-\frac{1}{4} \sum\left[X, E_{\alpha}\right] E_{\alpha}, \\
M_{K} & =\sum \omega E_{i} \chi_{K}\left(E_{i}\right), \\
A & =\sum \omega_{i} E_{i} \chi_{P}\left(E_{i}\right), \\
M & =\sum \omega E_{i} \chi\left(E_{i}\right)+\sum \omega E_{\alpha} \chi\left(E_{\alpha}\right) .
\end{aligned}
$$


Clearly $\chi(X)=\chi_{K}(X)+\chi_{P}(X)$ and $\chi_{K}$ is the spin representation of $\mathscr{K}$ extended to act on $S$. If the isotropy representation $K \rightarrow \operatorname{SO}(\mathscr{P})$ lifts to spin then this induces $\chi_{P} \mid \mathscr{K}$, see Lemma 2.1 of [5].

LEMMA 2.2. $M=M_{K}+3 A$.

Proof. Observe that

$$
\sum_{\gamma} E_{\gamma}\left[E_{\gamma}, E_{\alpha}\right]=\sum_{i} E_{i}\left[E_{i}, E_{\alpha}\right]
$$

since

$$
\begin{aligned}
\sum E_{\gamma}\left[E_{\gamma}, E_{\alpha}\right] & =\sum E_{\gamma}\left\langle\left[E_{\gamma}, E_{\alpha}\right], E_{i}\right\rangle E_{i}=\sum-E_{\gamma}\left\langle\left[E_{i}, E_{\alpha}\right], E_{\gamma}\right\rangle E \\
& =\sum-\left[E_{i}, E_{\alpha}\right] E_{i}=\sum E_{i}\left[E_{i}, E_{\alpha}\right] .
\end{aligned}
$$

The result now follows.

Proposition 2.3. $P_{t} \psi^{t}=\frac{1}{2 t} M_{K} \psi+\frac{1}{2}\left(\frac{2}{t}+t\right) A \psi$.

Proof. We calculate:

$$
\text { (2.9) } \begin{aligned}
P_{t} \psi^{t}= & -\frac{1}{4} \sum \frac{1}{t} \omega E_{i}\left(\nabla_{E_{t}} E_{j}\right) E_{j} \psi \\
& -\frac{1}{4} \sum\left(\frac{2}{t}-t\right) \omega E_{i}\left(\nabla_{E_{t}} E_{\beta}\right) E_{\beta} \psi \\
& -\frac{1}{4} \sum t \omega E_{\alpha}\left(\nabla_{E_{\alpha}} E_{\beta}\right) E_{\beta} \psi-\frac{1}{4} \sum t \omega E_{\alpha}\left(\nabla_{E_{\alpha}} E_{\beta}\right) E_{\beta} \psi \\
= & -\frac{1}{8} \sum \frac{1}{t} \omega E_{i}\left[E_{i}, E_{j}\right] E_{j} \psi \\
& -\frac{1}{8} \sum\left(\frac{2}{t}-t\right) \omega E_{i}\left[E_{i}, E_{\beta}\right] E_{\beta} \psi \\
& -\frac{1}{8} \sum t \omega E_{\alpha}\left[E_{\alpha}, E_{\beta}\right] E_{\beta} \psi-\frac{1}{8} \sum t \omega E_{\alpha}\left[E_{\alpha}, E_{\beta}\right] E_{\beta} \psi \\
= & \frac{1}{2 t} M_{K} \psi+\frac{1}{2}\left(\frac{2}{t}+t\right) A \psi,
\end{aligned}
$$

which is the result of the proposition.

COROllary 2.4 .

$$
P_{t}=1 / t Q_{K}+Q_{P}+1 / 2 t\left(1 \otimes M_{K}\right)+(1 / t+t / 2)(1 \otimes A) .
$$

LEMma 2.5. The operators $Q_{K}, Q_{P}, 1 \otimes M_{K}, 1 \otimes A$ and hence $P_{t}$ all commute with the action of $\mathscr{K}$ via the representation $\nu \otimes \chi$. 
Proof. This is another direct calculation. For example in the case of $Q_{K}$ :

(2.10) $\left[Q_{K},(\nu \otimes 1+1 \otimes \chi) E_{i}\right]$

$$
\begin{aligned}
= & \sum \nu\left(\left[E_{j}, E_{i}\right]\right) \otimes \omega E_{j} \\
& \quad+\sum \nu\left(E_{j}\right) \otimes \omega\left(E_{j} \chi\left(E_{i}\right)-\chi\left(E_{i}\right) E_{j}\right) \\
= & \sum \nu\left(\left[E_{j}, E_{i}\right]\right) \otimes \omega E_{j}+\sum \nu\left(E_{j}\right) \otimes \omega\left[E_{j}, E_{i}\right]=0 .
\end{aligned}
$$

Proposition 2.6. The operator $P_{t}$ preserves the decomposition $\Gamma(\underset{\sim}{S})$ $=L^{2}(\Gamma \backslash G) \otimes S=\widehat{\oplus} V_{\lambda} \otimes S$ into isotypic components under the right regular representation $\nu \otimes 1$ of $G$.

Proof. This is immediate since

$$
P_{t}=Q_{t}+(1 / 2 t) 1 \otimes M_{K}+(1 / t+t / 2) 1 \otimes A
$$

and $Q_{t}$ is a linear combination of the operators $\nu(E)$.

Let $\Omega_{G}=-\sum E_{i}^{2}-\sum E_{\alpha}^{2}$ and $\Omega_{K}=-\sum E_{i}^{2}$ be the Casimir elements. Set $\Omega_{P}=\Omega_{G}-\Omega_{K}$ and let $\rho_{K}$ denote half the sum of the positive roots of $K$. Then define the following operators:

$$
\begin{aligned}
\text { (i) } & R_{K}=\sum \nu\left(E_{i}\right) \otimes \chi_{K}\left(E_{i}\right), \\
\text { (ii) } & R_{P}=\sum \nu\left(E_{\alpha}\right) \otimes \chi_{P}\left(E_{\alpha}\right), \\
\text { (iii) } & R_{M}=\sum \nu\left(E_{i}\right) \otimes \chi_{P}\left(E_{i}\right), \\
\text { (iv) } & R_{S}=\sum \chi_{K}\left(E_{i}\right) \chi_{P}\left(E_{i}\right),
\end{aligned}
$$

where $\chi_{K}$ and $\chi_{P}$ are given in (2.7). Notice that $R_{S}$ is an operator on $S$ while the other three operate on $C^{\infty}(G) \otimes S$. Direct calculation now establishes the following result.

Proposition 2.7. Using the notation $\{U, V\}=U V+V U$ :

(i) $\left\{Q_{K}, Q_{P}\right\}=4 R_{P}$,

(ii) $\left\{Q_{K}, 1 \otimes M_{K}\right\}=-6 R_{K}$,

(iii) $\left\{Q_{K}, 1 \otimes A\right\}=-2 R_{M}$,

(iv) $\left\{Q_{P}, 1 \otimes M_{K}\right\}=0$,

(v) $\left\{Q_{P}, 1 \otimes A\right\}=-4 R_{P}$,

(vi) $\left\{M_{K}, A\right\}=-6 R_{S}$,

(vii) $Q_{K}^{2}=\nu\left(\Omega_{K}\right) \otimes 1+2 R_{K}$,

(viii) $Q_{P}^{2}=\nu\left(\Omega_{P}\right) \otimes 1+2 R_{M}$, 

(ix) $A^{2}=\chi_{P}\left(\Omega_{K}\right)+2 R_{S}$,
(x) $M_{K}^{2}=9\left\|\rho_{K}\right\|^{2}$.

Proof. To illustrate the proof, we verify part (x):

$$
\text { (2.12) } \begin{aligned}
M_{K}^{2}= & \sum \omega E_{i} \chi_{K}\left(E_{i}\right) \omega E_{j} \chi_{K}\left(E_{j}\right)=\sum E_{i} \chi_{K}\left(E_{i}\right) E_{j} \chi_{K}\left(E_{j}\right) \\
= & \frac{1}{2} \sum\left(E_{i} E_{j} \chi_{K}\left(E_{i}\right) \chi_{K}\left(E_{j}\right)+E_{j} E_{i} \chi_{K}\left(E_{j}\right) \chi_{K}\left(E_{i}\right)\right. \\
& \left.+E_{i}\left[E_{i}, E_{j}\right] \chi_{K}\left(E_{j}\right)\right) \\
= & \frac{1}{2}\left\{\sum E_{i} E_{j}\left(\chi_{K}\left(E_{i}\right) \chi_{K}\left(E_{j}\right)-\chi_{K}\left(E_{j}\right) \chi_{K}\left(E_{i}\right)\right)\right. \\
& \left.-2 \sum \chi_{K}\left(E_{i}\right)^{2}\right\} \\
= & \frac{1}{2} \sum E_{i} E_{j} \chi_{K}\left(\left[E_{i}, E_{j}\right]\right)-5 \sum \chi_{K}\left(E_{i}\right)^{2} .
\end{aligned}
$$

Now

$$
\begin{gathered}
\sum E_{i} E_{j} \chi_{K}\left(\left[E_{i}, E_{j}\right]\right)=-\sum\left[E_{s}, E_{j}\right] E_{j} \chi_{K}\left(E_{s}\right) \\
=4 \sum \chi_{K}\left(E_{s}\right)^{2}=-4 \chi_{K}\left(\Omega_{K}\right) .
\end{gathered}
$$

Thus $M_{K}^{2}=3 \chi_{K}\left(\Omega_{K}\right)=9\left\|\rho_{K}\right\|^{2}$, since $\chi_{K}$ is the sum of irreducible representations, each taking the same value, $3\left\|\rho_{K}\right\|^{2}$, on $\Omega_{K}$.

The space of sections $\Gamma \underset{\sim}{S})$ has been decomposed into a completed sum of terms of the form $V_{\lambda} \otimes S, \lambda \in \widehat{G}$, under the action of the group $G$. Each $V_{\lambda}$ is finite-dimensional and we may decompose $V_{\lambda} \otimes S$ under the $\nu \otimes \chi$ action of $\mathscr{K}$ into isotypic (rather than irreducible) components:

$$
V_{\lambda} \otimes S=\bigoplus S_{\theta} .
$$

Now Lemma 2.5 and Proposition 2.6 tell us that $P_{t}$ leaves $S_{\theta}$ invariant. The next step is to show $P_{t}^{2}$ is constant on $S_{\theta}$ and then that $\operatorname{tr} P_{t} \mid S_{\theta}=0$. To show $P_{t}^{2} \mid S_{\theta}$ is constant we show that each of the ten operators of Proposition 2.7 is constant on $S_{\theta}$. This is clearly the same as showing $R_{K}, R_{P}, R_{M}$ and $R_{S}$ are constant on $S_{\theta}$.

LEMMA 2.8. The operators $R_{K}, R_{P}, R_{M}$ and $R_{S}$ are constants on $S_{\theta}$.

Proof. First notice that while $\chi_{K}$ and $\chi_{P}$ may not be irreducible the Casimir takes the same value in each irreducible summand, see 
[5, Lemma 2.2]. The result, for all except $R_{S}$, now follows from the following formulae:

$$
\begin{aligned}
R_{K} & =\frac{1}{2}\left(-\nu \otimes \chi_{K}\left(\Omega_{K}\right)+\nu\left(\Omega_{K}\right) \otimes 1+1 \otimes \chi_{K}\left(\Omega_{K}\right)\right), \\
R_{P} & =\frac{1}{2}\left(-\nu \otimes \chi_{P}\left(\Omega_{P}\right)+\nu\left(\Omega_{P}\right) \otimes 1+1 \otimes \chi_{P}\left(\Omega_{P}\right)\right), \\
R_{M} & =\frac{1}{2}\left(-\nu \otimes \chi_{P}\left(\Omega_{K}\right)+\nu\left(\Omega_{K}\right) \otimes 1+1 \otimes \chi_{P}\left(\Omega_{K}\right)\right) .
\end{aligned}
$$

For $R_{S}$ consider the decomposition $\mathscr{G}=\mathscr{K} \otimes \mathscr{P}$. It gives rise to an isomorphism $\operatorname{Cliff}(\mathscr{G}) \cong \operatorname{Cliff}(\mathscr{K}) \otimes \operatorname{Cliff}(\mathscr{P})$ and thence to one of modules:

$$
S \cong S_{K} \otimes S_{P}
$$

With respect to this decomposition $\chi_{K}=\widehat{\chi}_{K} \otimes 1$ and $\chi_{P}=1 \otimes \widehat{\chi}_{P}$ so that

$$
R_{S}=\frac{1}{2}\left(-\widehat{\chi}_{K} \otimes \widehat{\chi}_{P}\left(\Omega_{K}\right)+\widehat{\chi}_{K} \otimes 1\left(\Omega_{K}\right)+1 \otimes \widehat{\chi}_{P}\left(\Omega_{K}\right)\right) .
$$

COROLlaRY 2.9. The operator $P_{t}^{2} \mid S_{\theta}$ is constant.

This constant depends on $t$ and $\theta$. In principle it has been calculated but is omitted as the expression is unenlightening.

Proposition 2.10. If $\operatorname{rank} G>1, \operatorname{tr} P_{t} \mid S_{\theta}=0$.

Proof. Let $U_{p}$ be the subspace of $\operatorname{Cliff}(\mathscr{G})$ spanned as a vector space by $E_{i_{1}} E_{i_{2}} \cdots E_{i_{p}}, i_{1}<i_{2}<\cdots<i_{p}$ (this time without using the convention of Latin and Greek indices). Then for $X \in U_{p}$, we have

$$
\operatorname{tr} X=0 \text { for } p \neq 0 \text {. }
$$

Since $M_{K}=\sum \omega E_{i} \chi_{K}\left(E_{i}\right)=\frac{1}{4} \sum \omega E_{i}\left[E_{i}, E_{j}\right] E_{j}$ and $\operatorname{rank} G>1$ (so $\operatorname{dim} \mathscr{G}>3$ ) it is clear that $M_{K} \in U_{r+s-3}$. Thus by equation (2.18), since $r+s>3, \operatorname{tr} M_{K} \mid S=0$. Split $S$ into eigenspaces of $M_{K}: S=\left(S_{K}^{+} \oplus S_{K}^{-}\right) \otimes S_{P}=\left(S_{K}^{+} \otimes S_{P}\right) \oplus\left(S_{K}^{-} \otimes S_{P}\right)$. Since $M_{K}^{2}=\alpha^{2}$, $\alpha=3\left\|\rho_{K}\right\|$, there are only two eigenspaces and $\operatorname{tr} M_{K}=0$ gives $\operatorname{dim} S_{K}^{+}=\operatorname{dim} S_{K}^{-}$. By considering weights $S_{K} \cong 2^{n} V_{\rho_{K}}, n=\frac{1}{2}(l-1)$, so that $S_{K}^{+} \cong S_{K}^{-} \cong 2^{n-1} V_{\rho_{K}}$ and $S_{\theta}=S_{\theta}^{+} \oplus S_{\theta}^{-}$with $\operatorname{dim} S_{\theta}^{+}=$ $\operatorname{dim} S_{\theta}^{-}$. Thus $\operatorname{tr} M_{K} \mid S_{\theta}=0$ and with respect to the decomposition $M_{K}$ has matrix $\left(\begin{array}{cc}\alpha & 0 \\ 0 & -\alpha\end{array}\right)$. If $B$ is any operator with matrix $\left(\begin{array}{ll}u & v \\ x & y\end{array}\right)$ then $\left\{M_{K}, B\right\}=\left(\begin{array}{cc}\alpha u & 0 \\ 0 & -2 \alpha y\end{array}\right)$. Thus if $\left\{M_{K}, B\right\}$ is constant on $S_{\theta}$ then $u=-y$ and $\operatorname{tr} B \mid S_{\theta}=0$. Taking $B=Q_{K}, Q_{P}$ and $A$ we see $\operatorname{tr} Q_{K}\left|S_{\theta}=\operatorname{tr} Q_{P}\right| S_{\theta}=\operatorname{tr} A \mid S_{\theta}=0$. Hence $\operatorname{tr} P_{t} \mid S_{\theta}=0$. 
THeOREM 2.11. $P_{t}$ has spectral symmetry for all $t>0$ if $\operatorname{rank} G$ $>1$.

THEOREM 2.12. The equivariant eta function of the operator $P_{t}$ on $\Gamma \backslash G$, for rank $G>1$ at $t>0$ and any discrete co-compact subgroup $\Gamma$ vanishes as a K-character: $\eta_{K}(s, g)=0$ where $\eta_{K}(s, g)=$ $\sum_{\lambda} \operatorname{sign}(\lambda)|\lambda|^{-s} \operatorname{tr}\left(g \mid V_{\lambda}\right)$ for $g \in K$.

3. Spectral symmetry for a symmetric pair of noncompact type. Let $(G, K)$ be a symmetric pair of noncompact type. This case is similar to that of the previous section. However, the details are different and we shall be concerned, mainly, with pointing out the differences. Decompose $\mathscr{G}=\mathscr{K} \oplus \mathscr{P}$ and define the metric $\rho$ to be the negative of the Killing form on $\mathscr{K}$, the Killing form on $\mathscr{P}$ and under $\rho$ let $\mathscr{K}$ be orthogonal to $\mathscr{P}$. As before let $E_{1}, \ldots, E_{r}$ be an orthonormal basis for $\mathscr{K} ; E_{r+1}, \ldots, E_{r+s}$ be one for $\mathscr{P}$ and we shall use the convention that Latin subscripts run from 1 to $r$ and Greek from $r+1$ to $r+s$. Set $e_{i}=E_{i} / t, e_{\alpha}=E_{\alpha}$ and let $\rho_{t}$ be the metric with $e_{1}, \ldots, e_{r+s}$ as orthonormal basis. Let $\chi_{K}, \chi_{P}, Q_{K}, Q_{P}, M_{K}$ and $A$ be defined by the formulae of the previous section.

Formally we can use the compact dual $\mathscr{G}^{*}$ of $\mathscr{G}$ to obtain the present results from the previous section. Let $\mathscr{G}_{\mathbf{C}}$ be the complexification of $\mathscr{G}$. Then there is the compact dual $\mathscr{G}^{*} \subset \mathscr{G}_{\mathbf{C}}$ of $\mathscr{G}$ and a correspondence

$$
X \rightarrow X \quad \text { for } X \in \mathscr{K}, \quad X \rightarrow i X \quad \text { for } X \in \mathscr{P} \quad(i=\sqrt{-1})
$$

between $\mathscr{G}$ and $\mathscr{G}^{*}$. Denote by $X^{*}$ the element of $\mathscr{G}^{*}$ corresponding to $X \in \mathscr{G}$ so $e_{j}^{*}=e_{j}$ and $e_{\alpha}^{*}=i e_{\alpha}$. There is a metric $\rho_{t}^{*}$ on $\mathscr{G}^{*}$ with orthonormal basis $e_{1}^{*}, \ldots, e_{r+s}^{*}$. Formally

$$
\rho_{t}(x, y)=\rho_{i t}^{*}\left(i x^{*}, i y^{*}\right)
$$

and so as elements of the Lie algebra one is led to expect

$$
P_{t} \psi^{t}=i P_{i t}^{*} \psi^{t *}
$$

In fact this is true as a direct, rather than formal, calculation shows.

Proposition 3.1. $P_{t} \psi^{t}=\frac{1}{2 t} M_{K} \psi+\frac{1}{2}\left(\frac{2}{t}-t\right) A \psi$.

Proof. This is essentially the same as the proof of Proposition 2.3. The main changes are as follows. Firstly the invariance of the metric 
is now given by

$$
\begin{aligned}
& \left\langle E_{\beta},\left[E_{i}, E_{\gamma}\right]\right\rangle=-\left\langle E_{\gamma},\left[E_{i}, E_{\beta}\right]\right\rangle, \\
& \left\langle E_{i},\left[E_{\beta}, E_{\gamma}\right]\right\rangle=+\left\langle E_{\gamma},\left[E_{\beta}, E_{i}\right]\right\rangle
\end{aligned}
$$

instead of always a negative sign. Thus

$$
\sum E_{\gamma}\left[E_{\gamma}, E_{\alpha}\right]=-\sum E_{i}\left[E_{i}, E_{\alpha}\right]
$$

and so

$$
A=-\frac{1}{4} \sum \omega E_{i}\left[E_{i}, E_{\alpha}\right] E_{\alpha}=\frac{1}{4} \sum \omega E_{\alpha}\left[E_{\alpha}, E_{\beta}\right] E_{\beta} .
$$

The formula $\nabla_{X} Y=1 / 2[X, Y]$ no longer holds for all $X$ and $Y$. Instead we have

$$
\begin{array}{ll}
\nabla_{E_{l}} E_{j}=\frac{1}{2}\left[E_{i}, E_{j}\right], & \nabla_{E_{l}} E_{\beta}=\frac{3}{2}\left[E_{i}, E_{\beta}\right], \\
\nabla_{E_{\alpha}} E_{j}=-\frac{1}{2}\left[E_{\alpha}, E_{j}\right], & \nabla_{E_{\alpha}} E_{\beta}=\frac{1}{2}\left[E_{\alpha}, E_{\beta}\right] .
\end{array}
$$

Then equations $(2.5)$ in the noncompact case become

$$
\begin{aligned}
& \text { (i) }\left\langle\nabla_{e_{t}}^{t} e_{j}, e_{k}\right\rangle_{t}=\frac{1}{t}\left\langle\nabla_{E_{t}} E_{j}, E_{k}\right\rangle, \\
& \text { (ii) }\left\langle\nabla_{e_{t}}^{t} e_{\beta}, e_{\gamma}\right\rangle_{t}=\frac{1}{3}\left(\frac{2}{t}+t\right)\left\langle\nabla_{E_{t}} E_{\beta}, E_{\gamma}\right\rangle, \\
& \text { (iii) }\left\langle\nabla_{e_{\alpha}}^{t} e_{j}, e_{\gamma}\right\rangle_{t}=t\left\langle\nabla_{E_{\alpha}} E_{j}, E_{\gamma}\right\rangle, \\
& \text { (iv) }\left\langle\nabla_{e_{\alpha}}^{t} e_{\beta}, e_{k}\right\rangle_{t}=t\left\langle\nabla_{E_{\alpha}} E_{\beta}, E_{k}\right\rangle .
\end{aligned}
$$

As before the other expressions analogous to these with an odd number of Greek indices are zero. The result of Proposition 2.1 is now:

$$
\begin{aligned}
\text { (i) } \nabla_{e_{t}}^{t} e_{j} & =\left(1 / t^{2}\right) \nabla_{E_{t}} E_{j}, \\
\text { (ii) } \nabla_{e_{t}}^{t} e_{\beta} & =\frac{1}{3}\left(\frac{2}{t}+t\right) \nabla_{E_{t}} E_{\beta}, \\
\text { (iii) } \nabla_{e_{\alpha}} e_{j} & =t \nabla_{E_{\alpha}} E_{j}, \\
\text { (iv) } \nabla_{e_{\alpha}}^{t} e_{\beta} & =\nabla_{E_{\alpha}} E_{\beta} .
\end{aligned}
$$

The proof is completed by a calculation similar to that used to prove Proposition 2.3.

The list of relations in Proposition 2.7 takes the following form where the operators $R_{K}, R_{P}, R_{M}$ and $R_{S}$ are defined by the formulae (2.11). 
Proposition 3.2.

(i) $\left\{Q_{K}, Q_{P}\right\}=-4 R_{P}$,

(ii) $\left\{Q_{K}, 1 \otimes M_{K}\right\}=-6 R_{K}$,

(iii) $\left\{Q_{K}, 1 \otimes A\right\}=-2 R_{M}$,

(iv) $\left\{Q_{P}, 1 \otimes M_{K}\right\}=0$,

(v) $\left\{Q_{P}, 1 \otimes A\right\}=4 R_{P}$,

(vi) $\left\{M_{K}, A\right\}=-6 R_{S}$,

(vii) $Q_{K}^{2}=\nu\left(\Omega_{K}\right) \otimes 1+2 R_{K}$,

(viii) $Q_{P}^{2}=\nu\left(\Omega_{P}\right) \otimes 1-2 R_{M}$,

(ix) $A^{2}=\chi_{P}\left(\Omega_{K}\right) \otimes 1+2 R_{S}$,

(x) $M_{K}^{2}=9\left\|\rho_{K}\right\|^{2}$.

Now let $\Gamma$ be any co-compact discrete subgroup of $G$. Then the space of $L^{2}$-sections of the spin bundle $\underset{\sim}{S}$ over $\Gamma \backslash G$ decomposes into a completed sum of unitary representations of $G$. For $\lambda \in \widehat{G}$ let $V_{\lambda}^{\Gamma}$ be the isotypic summand of type $\lambda$ so that

$$
L^{2}(\underset{\sim}{S})=\widehat{\bigoplus} V_{\lambda}^{\Gamma} \otimes S .
$$

The representations $\lambda$ with $V_{\lambda}^{\Gamma} \neq 0$ occurring in this sum are, in general, not explicitly known. Each term in this sum decomposes further into $\mathscr{K}$-types under the action $\nu \otimes \chi$ :

$$
V_{\lambda}^{\Gamma} \otimes S=\bigoplus S_{\theta}
$$

The arguments of $\S 2$ go through word for word. So there is spectral symmetry for $P_{t}$ on each $S_{\theta}$ providing rank $G>1$. Consequently we have the following theorem.

THEOREM 3.3. The equivariant eta function for the operator $P_{t}$ on $\Gamma \backslash G$ vanishes as a $K$-character for $G$ a real semi-simple Lie group of rank $>1$ and $\Gamma$ a co-compact discrete subgroup.

\section{REFERENCES}

[1] M. F. Atiyah, V. K. Patodi, and I. M. Singer, Spectral asymmetry and Rie-s mannian geometry I and II, Math. Proc. Camb. Phil. Soc., 77 (1975), 43-69and 78 (1976), 405-432.

[2] H. D. Fegan and B. Steer, On the "strange formula" of Freudenthal and de Vries, Math. Proc. Camb. Phil. Soc., 105 (1989), 249-252.

[3] C. S. Gordon, Naturally reductive homogeneous Riemannian manifolds, Canad. J. Math., 37 (1985), 467-487. 
[4] N. Hitchin, Harmonic spinors, Advances in Math., 14 (1974), 1-55.

[5] R. Parthasarathy, Dirac operators and the discrete series, Ann. of Math., 96 (1972), 1-30.

[6] J. A. Seade and B. Steer, $A$ note on the eta-function for quotients of $\mathrm{PSL}_{2}(\mathbf{R})$ by co-compact Fuchsian groups, Topology, 26 (1987), 79-91.

Received December 5, 1988 and in revised form September 1, 1989.

The UNIVERSity OF NeW MeXico

AlbuQUeRQue, NM 87131

AND

HertFord COLlEge

OXFORD OX1 3BW, ENGLAND 



\title{
PACIFIC JOURNAL OF MATHEMATICS EDITORS
}

\author{
V. S. VARADARAJAN \\ (Managing Editor) \\ University of California \\ Los Angeles, CA 90024-1555-05 \\ Herbert Clemens \\ University of Utah \\ Salt Lake City, UT 84112 \\ ThOMAS ENRIGHT \\ University of California, San Diego \\ La Jolla, CA 92093
}

R. FINN

Stanford University

Stanford, CA 94305

HeRmanN FlaschKa

University of Arizona

Tucson, AZ 85721

VAUGHaN F. R. Jones

University of California

Berkeley, CA 94720

Steven Kerckhoff

Stanford University

Stanford, CA 94305

\section{C. MOORE}

University of California

Berkeley, CA 94720

MaRTin SCHARLEMANN

University of California

Santa Barbara, CA 93106

HAROLD STARK

University of California, San Diego

La Jolla, CA 92093

\section{ASSOCIATE EDITORS}
R. ARENS
E. F. BECKENBACH (1906-1982)
B. H. NeumanN
F. WOLF
(1904-1989)
K. YosHidA

\section{SUPPORTING INSTITUTIONS}

UNIVERSITY OF ARIZONA
UNIVERSITY OF BRITISH COLUMBIA
CALIFORNIA INSTITUTE OF TECHNOLOGY
UNIVERSITY OF CALIFORNIA
MONTANA STATE UNIVERSITY
UNIVERSITY OF NEVADA, RENO
NEW MEXICO STATE UNIVERSITY
OREGON STATE UNIVERSITY
UNIVERSITY OF ARIZONA
UNIVERSITY OF BRITISH COLUMBIA UNIVERSITY OF CALIFORNIA
MONTANA STATE UNIVERSITY
NEW MEXICO STATE UNIVERSITY

\author{
UNIVERSITY OF SOUTHERN CALIFORNIA \\ STANFORD UNIVERSITY \\ UNIVERSITY OF HAWAII \\ UNIVERSITY OF TOKYO \\ UNIVERSITY OF UTAH \\ WASHINGTON STATE UNIVERSITY \\ UNIVERSITY OF WASHINGTON
}
UNIVERSITY OF OREGON

The Supporting Institutions listed above contribute to the cost of publication of this Journal, but they are not owners or publishers and have no responsibility for its content or policies.

\begin{abstract}
Mathematical papers intended for publication in the Pacific Journal of Mathematics should be in typed form or offset-reproduced (not dittoed), double spaced with large margins. Please do not use built up fractions in the text of the manuscript. However, you may use them in the displayed equations. Underline Greek letters in red, German in green, and script in blue. The first paragraph must be capable of being used separately as a synopsis of the entire paper. In particular it should contain no bibliographic references. Please propose a heading for the odd numbered pages of less than 35 characters. Manuscripts, in triplicate, may be sent to any one of the editors. Please classify according to the 1980 Mathematics Subject Classification (1985 Revision) scheme which can be found in the December index volumes of Mathematical Reviews. Supply name and address of author to whom proofs should be sent. All other communications should be addressed to the managing editor, or Elaine Barth, University of California, Los Angeles, California 90024-1555-05.

There are page-charges associated with articles appearing in the Pacific Journal of Mathematics. These charges are expected to be paid by the author's University, Government Agency or Company. If the author or authors do not have access to such Institutional support these charges are waived. Single authors will receive 50 free reprints; joint authors will receive a total of 100 free reprints. Additional copies may be obtained at cost in multiples of 50 .
\end{abstract}

The Pacific Journal of Mathematics (ISSN 0030-8730) is published monthly. Regular subscription rate: $\$ 190.00$ a year (12 issues). Special rate: $\$ 95.00$ a year to individual members of supporting institutions.

Subscriptions, orders for numbers issued in the last three calendar years, and changes of address should be sent to Pacific Journal of Mathematics, P.O. Box 969, Carmel Valley, CA 93924, U.S.A. Old back numbers obtainable from Kraus Periodicals Co., Route 100, Millwood, NY 10546.

The Pacific Journal of Mathematics at P.O. Box 969, Carmel Valley, CA 93924 (ISSN 0030-8730) is published monthly. Second-class postage paid at Carmel Valley, California 93924, and additional mailing offices. Postmaster: send address changes to Pacific Journal of Mathematics, P.O. Box 969, Carmel Valley, CA 93924.

\section{PUBLISHED BY PACIFIC JOURNAL OF MATHEMATICS, A NON-PROFIT CORPORATION}




\section{Pacific Journal of Mathematics}

Vol. 145, No. $2 \quad$ October, 1990

Chong Hsio Fang and Minking Eie, On the values of a zeta function at

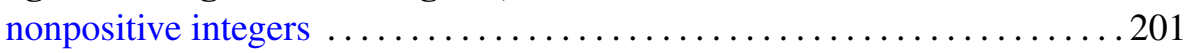

Howard D. Fegan, Brian F. Steer and L. Whiteway, Spectral symmetry of the Dirac operator for compact and noncompact symmetric pairs ..... 211

William James Heinzer and David C. Lantz, Integral domains that lose

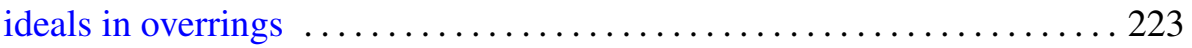

Alexander Eben Koonce, Relations among generalized characteristic classes .......................................239

M. S. Narasimhan and Günther Trautmann, Compactification of

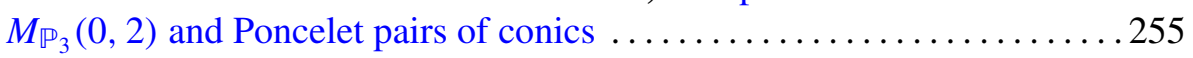

James Alexander Reeds, III and Lawrence A. Shepp, Optimal paths for a car that goes both forwards and backwards . ................. 367

Ai-Nung Wang, Constant mean curvature surfaces on a strip ............ 395 